\title{
Spin-size effects in stochastic resonance in uniaxial superparamagnets
}

\author{
Yuri P. Kalmykov, ${ }^{1}$ Serguey V. Titov, ${ }^{2}$ and William T. Coffey ${ }^{3}$ \\ ${ }^{1}$ Laboratoire de Mathématiques, Physique et Systèmes, Université de Perpignan Via Domitia, 52 Avenue de Paul Alduy, \\ 66860 Perpignan Cedex, France \\ ${ }^{2}$ Institute of Radio Engineering and Electronics of RAS, 1 Vvedenskii Square, Fryazino, Moscow Region 141190, Russia \\ ${ }^{3}$ Department of Electronic and Electrical Engineering, Trinity College, Dublin 2, Ireland
}

(Received 20 April 2010; published 28 May 2010)

\begin{abstract}
The relaxation dynamics of a uniaxial paramagnet of arbitrary spin value $S$, interacting with a thermal bath, are treated using the spin-boson model for a particular spin Hamiltonian consisting of the simplest uniaxial potential in the high temperature, Ohmic damping, and weak spin-bath coupling limits. The signal-to-noise ratio of the magnetic moment fluctuations in the stochastic resonance of such a paramagnet displays a pronounced dependence on $S$ for values of $S<20$. The dependence arises from the quantum spin dynamics which differs markedly from the magnetization dynamics of classical superparamagnets with $S \sim 10^{3}-10^{5}$.
\end{abstract}

DOI: $10.1103 /$ PhysRevB.81.172411

PACS number(s): 75.20.-g, 05.40.Ca, 75.50.Tt, 75.50.Xx

A particularly interesting aspect of the motion of Brownian particles in a multiwell potential is the phenomenon of stochastic resonance (SR), whereby a weak periodic forcing synchronized with the thermally activated hopping between the potential wells greatly enhances the rate of switching between them. The archetypal theoretical model of SR (Ref. 1) is a Brownian particle in a bistable potential subjected to noise arising from the thermal bath. The particle is excited by a weak ac driving force of frequency $\Omega$ close to the rate of transitions (escape rates) between the wells. However, the amplitude of the force is in itself insufficient to induce the transitions. Consequently, switching may occur only by the combined effect of the deterministic ac force and the noise. The resulting signal-to-noise ratio (SNR) as a function of the noise intensity has a bell-like shape, i.e., it passes through a maximum thus exhibiting stochastic resonance due to the ability of noise to enhance the intensity of the interwell hoppings in the system. Stochastic resonance is now well known but still remarkable effect allowing one to control the behavior of periodic signals passing through noisy systems and is a universal manifestation of the interplay between stochastic and regular motions. Comprehensive reviews of diverse aspects of SR are available in Refs. 1-3.

The behavior of magnetic nanosystems (such as superparamagnetic particles, nanoclusters, and molecular magnets) forced by a weak ac magnetic field is yet another important manifestation of SR. Here the magnetic anisotropy provides the multistable states for the magnetization while the thermal fluctuations due to the bath are the source of the noise. These conditions give rise to magnetic stochastic resonance which may be defined as the enhancement of the SNR of the magnetic moment fluctuations due to noise. ${ }^{4}$ The SNR of the magnetic moment fluctuations is of interest because nanoparticle magnetism is a rapidly expanding area of research with many applications. These arise both in the (applied) area of information storage and in other (fundamental) aspects such as the crossover between classical and quantum behavior of the magnetization since single-domain particles exhibit essentially classical behavior while smaller entities such as nanoclusters made of many atoms and molecular magnets exhibit pronounced quantum behavior.

The main features of the magnetic SR are clearly mani- fested by uniaxial single-domain particles ${ }^{5-13}$ and may be completely understood in terms of the classical (macrospin) model of the coherent rotation of the magnetization. ${ }^{14}$ Here each particle behaves like a huge paramagnetic atom having a magnetic moment $\sim 10^{4}-10^{5}$ Bohr magnetons, i.e., $S \sim 10^{4}-10^{5}$. Moreover, the magnetic free energy density $V$ is given by the symmetric bistable potential

$$
V(\vartheta)=-k T v^{-1} \sigma \cos ^{2} \vartheta
$$

where $\vartheta$ is the polar angle, $\sigma=v K /(k T)$ is the dimensionless barrier height parameter, $v$ is the volume of the particle, $k$ is Boltzmann's constant, $T$ is the absolute temperature, and $K$ is the anisotropy constant. In the absence of external magnetic fields, the magnetization of the uniaxial particle has two equivalent stable orientations at $\vartheta=0$ and $\vartheta=\pi$ so that it is an ideal example of a bistable system subjected to noise. Here the reversal of the classical spin is due to thermal activation. The rate of transitions between the potential wells is controlled by the parameter $\sigma$ so that one may regard $\sigma^{-1}$ as the dimensionless temperature, i.e., the noise intensity.

In contrast, we have little knowledge about magnetic SR in superparamagnets with smaller spin values $S \sim 10-100$ (such as molecular magnets and nanoclusters), where the spin reversal is either due to thermal activation or tunneling or a combination of both and quantum effects appear. These quantum effects differ from those in the SR for translational Brownian motion (see, e.g., Refs. 15 and 16 and references cited therein) because in spite of some analogies the quantum spin dynamics differ from those of Brownian particles. Here we shall treat quantum effects in the magnetic SR taking as an example a uniaxial paramagnet of arbitrary spin $S$ driven by a weak external probing ac field $\mathbf{H}(t)=\mathbf{H} \cos \Omega t$ applied along the $Z$ axis, i.e., the axis of symmetry Thus the Hamiltonian $\hat{H}$ has the form

$$
\hat{H}=-K S^{-2} \hat{S}_{Z}^{2}-\gamma \hbar H \cos (\Omega t) \hat{S}_{Z}+\hat{H}_{S B}+\hat{H}_{B},
$$

where $\hat{S}_{Z}$ is the $Z$ component of the spin operator $\mathbf{S}, \hbar$ is Planck's constant, and $\gamma$ is the gyromagnetic ratio. The term $\hat{H}_{S B}$ describes interaction of the spin with the thermostat, and 
$\hat{H}_{B}$ characterizes the thermostat. This Hamiltonian includes a uniaxial anisotropy term $\hat{H}_{S}=-K S^{-2} \hat{S}_{Z}^{2}$ exhibiting an energy spectrum $\varepsilon_{m}$ with a double-well structure and two minima at $m= \pm S$ and comprises a generic model for quantum relaxation phenomena in uniaxial spin systems such as molecular magnets, nanoclusters, etc. (see, e.g., Refs. 17 and 18). Now Garanin ${ }^{17}$ and also García-Palacios and Zueco ${ }^{18}$ using the spin density matrix in the second order of perturbation theory in the spin bath coupling have recently considered the longitudinal relaxation of quantum paramagnets for arbitrary $S$. They treated the quantum spin dynamics using the Hubbard operator representation of the evolution equation for the density matrix. The nonlinear relaxation of uniaxial spin systems has also been treated by Kalmykov et al. ${ }^{19}$ via the respective evolution equations for the reduced density matrix and corresponding phase-space quasiprobability distribution function using the methods already available for classical spins. ${ }^{13}$ For linear response, the quantum solutions ${ }^{19}$ agree with those given in Refs. 17 and 18 while in the large spin limit, their results reduce to those for classical uniaxial superparamagnets. ${ }^{13,20-22}$

Here we shall treat the spin size effects in the magnetic SR for uniaxial quantum paramagnets via Kubo's linear-response theory. ${ }^{23}$ According to linear-response theory, the normalized longitudinal dynamic susceptibility $\chi(\omega)=\chi^{\prime}(\omega)-i \chi^{\prime \prime}(\omega)$ of a quantum spin system is defined $\mathrm{as}^{23}$

$$
\chi(\omega) / \chi_{0}=1-i \omega \int_{0}^{\infty} C(t) e^{-i \omega t} d t
$$

where

$$
C(t)=\frac{\left\langle\int_{0}^{\beta} \hat{S}_{Z}(-i \lambda \hbar) \hat{S}_{Z}(t) d \lambda\right\rangle_{0}}{\left\langle\int_{0}^{\beta} \hat{S}_{Z}(-i \lambda \hbar) \hat{S}_{Z}(0) d \lambda\right\rangle_{0}}
$$

is the equilibrium correlation function, the brackets \langle\rangle$_{0}$ denote the equilibrium statistical average, $\beta=(k T)^{-1}$, and $\chi_{0}=(\gamma \hbar)^{2}\left\langle\int_{0}^{\beta} \hat{S}_{Z}(0) \hat{S}_{Z}(i \lambda \hbar) d \lambda\right\rangle_{0}$ is the static susceptibility. Furthermore, magnetic SR may be generally described using linear response theory as follows. A typical Fourier component $M_{\omega}$ of the longitudinal components of the magnetization of a spin system is related to the corresponding Fourier component of a weak applied ac field $H_{\omega}$ through $\chi(\omega)$, viz., $M_{\omega}=\chi(\omega) H_{\omega}$. The spectral density $\Phi_{M}^{(s)}(\Omega)$ of the forced magnetic oscillations in a field $H(t)=H \cos (\Omega t)$ at the excitation frequency $\Omega$ is $\Phi_{M}^{(s)}(\Omega)=[H|\chi(\Omega)|]^{2} / 2$ while the noiseinduced part $\Phi_{M}^{(n)}(\Omega)=\chi^{\prime \prime}(\Omega) /(\pi \beta \Omega)$ is obtained using the fluctuation-dissipation theorem. ${ }^{13}$ Thus on combining the above equations, one obtains the $\mathrm{SNR}=\Phi_{M}^{(s)} / \Phi_{M}^{(n)}$ of the magnetic moment fluctuations as $\mathrm{SNR}=\left[\pi(\beta \gamma \hbar H)^{2} /\left(2 \tau_{N} \sigma\right)\right] R_{\Omega}$, where the dimensionless SNR factor $R_{\Omega}$ is given by

$$
R_{\Omega}=\sigma \tau_{N} \Omega|\chi(\Omega)|^{2} /\left[\beta(\gamma \hbar)^{2} \chi^{\prime \prime}(\Omega)\right]
$$

$\sigma=\beta K$ and $\tau_{N}$ is a characteristic free diffusion time. ${ }^{19}$ The SNR factor $R_{\Omega}$ besides the obvious dependence on the noise intensity (temperature) and the frequency of the exciting field $\Omega$, as in the classical case, also depends on the spin value $S$. Equation (3) shows that the SNR is determined by the dynamic susceptibility, which is a fundamental dynamical characteristic of any relaxing system.

According to Eq. (2), the behavior of $\chi(\omega)$ in the frequency domain is completely determined by the time behavior of $C(t)$. For a uniaxial superparamagnet modeled by the Hamiltonian $\hat{H}$ given above, $C(t)$ may be written as the finite discrete set of relaxation modes ${ }^{18,19}$

$$
C(t)=\sum_{k=1}^{2 S} c_{k} e^{-\lambda_{k} t},
$$

where $\lambda_{k}$ are the eigenvalues of the (finite) system matrix and $\sum_{k=1}^{2 S} c_{k}=1$. Equations (2) and (4) allow us to formally write $\chi(\Omega)$ as the finite sum of Lorentzians

$$
\chi(\Omega)=\chi_{0} \sum_{k=1}^{2 S} \frac{c_{k}}{1+i \Omega / \lambda_{k}} .
$$

Consequently, the asymptotic behavior of $\chi(\Omega)$ in the extremes of very low and very high frequencies is given by

$$
\frac{\chi(\Omega)}{\chi_{0}} \sim \begin{cases}1-i \Omega \tau_{c o r}+\cdots, & \Omega \rightarrow 0, \\ -i\left(\Omega \tau_{e f}\right)^{-1}+\cdots, & \Omega \rightarrow \infty .\end{cases}
$$

where

$$
\tau_{c o r}=\sum_{k=1}^{2 S} c_{k} \lambda_{k}^{-1} \quad \text { and } \quad \tau_{e f}=\left(\sum_{k=1}^{2 S} c_{k} \lambda_{k}\right)^{-1} .
$$

Hence in the adiabatic $(\Omega \rightarrow 0)$ and very high-frequency $(\Omega \rightarrow \infty)$ limits, Eq. (3) can be simplified yielding

$$
R_{0}=\sigma \chi_{S} \tau_{N} / \tau_{c o r} \quad \text { and } \quad R_{\Omega \rightarrow \infty}=\sigma \chi_{S} \tau_{N} / \tau_{e f} .
$$

Here the relaxation times $\tau_{c o r}$ and $\tau_{e f}$ from Eq. (7) can be given by the analytic formulas ${ }^{19}$

$$
\begin{gathered}
\tau_{e f}=\frac{2 S^{2} \chi_{S} \tau_{N}}{\sum_{k=1-S}^{S}[S(S+1)-k(k-1)] \rho_{k}}, \\
\tau_{c o r}=\frac{2 \tau_{N} S^{2}}{\chi_{S}} \sum_{k=1-S}^{S} \frac{\left(\sum_{m=k}^{S} m \rho_{m}\right)^{2}}{[S(S+1)-k(k-1)] \rho_{k}},
\end{gathered}
$$

where $\chi_{S}=\sum_{m=-S}^{S} m^{2} \rho_{m}, \rho_{m}=e^{\sigma m^{2} / S^{2}} / Z_{S}$ are the matrix elements of the equilibrium density matrix, and $Z_{S}=\sum_{m=-S}^{S} \rho_{m}$ is the partition function.

In the low-frequency region $\left(\Omega / \lambda_{1} \leq 1\right)$, the evaluation of the SNR can be further simplified noting that two distinct bands appear in the spectrum of the imaginary part $\chi^{\prime \prime}(\Omega) .{ }^{18,19}$ The low-frequency band is due to the slowest ("interwell") relaxation mode. The characteristic frequency and the half-width of this band are determined by $\lambda_{1} \cdot{ }^{18,19}$ The high-frequency band in $\chi^{\prime \prime}(\Omega)$ is due to the individual near degenerate high-frequency modes corresponding to the ei- 


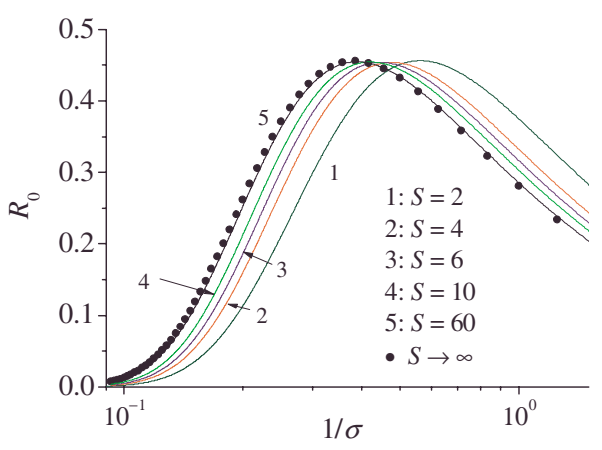

FIG. 1. (Color online) SNR of a uniaxial paramagnet in the adiabatic limit $\Omega=0$ as a function of dimensionless temperature $\sigma^{-1}$ for various $S$. Solid lines: the quantum Eq. (8). Filled circles: classical limit, $S \rightarrow \infty$ [Eq. (12)].

genvalues $\lambda_{k} \gg \lambda_{1}(k \geq 2)$. Thus, if one is interested solely in the low-frequency region, where their effect may be ignored, the dynamic susceptibility may be approximated as the single Lorentzian

$$
\frac{\chi(\Omega)}{\chi_{0}} \approx 1-\frac{i \Omega \tau_{c o r}}{1+i \Omega / \lambda_{1}} .
$$

The smallest eigenvalue $\lambda_{1}$ may be associated with the long time behavior of $C(t) \sim e^{-t / \tau}, t \gg \tau$, which is dominated by the longest relaxation (or the reversal) time of the magnetization $\tau=\lambda_{1}^{-1}$.

In the classical limit, $S \rightarrow \infty$, the SNR factor $R_{\Omega}$ is also given by Eq. (3). ${ }^{13}$ Thus the classical analogs of Eq. (8) are

$$
R_{0}=\sigma \tau_{N} \tau_{c o r}^{-1}\left\langle\cos ^{2} \vartheta\right\rangle_{0}, \quad R_{\Omega \rightarrow \infty}=\sigma \tau_{N} \tau_{e f}^{-1}\left\langle\cos ^{2} \vartheta\right\rangle_{0},
$$

where $\tau_{\text {cor }}$ and $\tau_{\text {ef }}$ can now be expressed in closed integral form as ${ }^{13,24}$

$$
\begin{gathered}
\tau_{\text {cor }}=\frac{2 \tau_{N}}{Z\left\langle\cos ^{2} \vartheta\right\rangle_{0}} \int_{-1}^{1}\left(\int_{-1}^{x} z e^{\sigma z^{2}} d z\right)^{2} \frac{e^{-\sigma x^{2}}}{1-x^{2}} d x, \\
\tau_{e f}=2 \tau_{N}\left\langle\cos ^{2} \vartheta\right\rangle_{0}\left(1-\left\langle\cos ^{2} \vartheta\right\rangle_{0}\right)^{-1},
\end{gathered}
$$

$Z=\int_{-1}^{1} e^{\sigma x^{2}} d x=\sqrt{\pi / \sigma} \operatorname{erf} i(\sqrt{\sigma})$ is the classical partition function, erf $i(x)=2 \pi^{1 / 2} \int_{0}^{x} e^{t^{2}} d t$ is the error function of imaginary argument, and

$$
\left\langle\cos ^{2} \vartheta\right\rangle_{0}=Z^{-1} \int_{-1}^{1} x^{2} e^{\sigma x^{2}} d x=e^{\sigma}(\sigma Z)^{-1}-(2 \sigma)^{-1} .
$$

The SNR in the adiabatic limit $\Omega=0, R_{\Omega=0}$, vs the dimensionless temperature parameter $\sigma^{-1}$ is shown in Fig. 1 for various values of $S$. The SNR curve $R_{0}$ has a bell-like shape thus exhibiting stochastic resonance, arising from the inherent ability of noise to enhance the intensity of the interwell hoppings of the magnetic moment. The maximum in $R_{0}$ is attained at $\sigma_{\max }^{-1} \sim 0.4 / 0.6$ and it shifts to low temperatures with increasing $S$ (for the molecular magnet $\mathrm{Mn}_{12}$ acetate with $S=10, \sigma_{\max }^{-1} \approx 0.45$ corresponds to $\left.T \sim 30 \mathrm{~K}\right)$. In the limit $\sigma^{-1} \rightarrow 0, R_{0} \rightarrow 0$. In general, we see that the quantum effects lead to both amplification and attenuation of the SNR

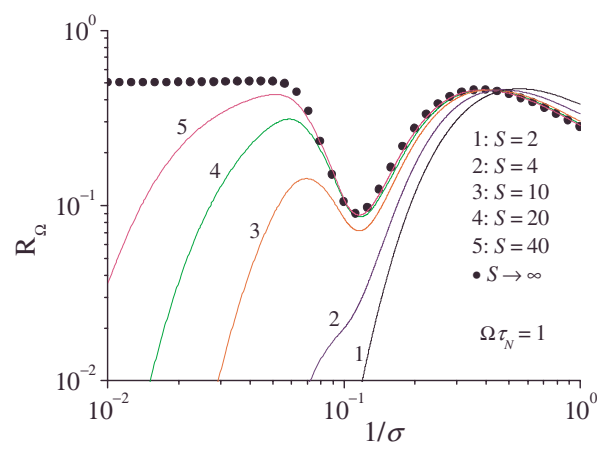

FIG. 2. (Color online) SNR as a function of $\sigma^{-1}$ for various values of $S$ and $\Omega \tau_{N}=1$. Solid lines: the quantum Eqs. (3) and (5). Filled circles: classical limit, $S \rightarrow \infty$.

(at high and low temperatures, respectively). The SNR function $R_{\Omega}$ vs $\sigma^{-1}$ at the finite frequency $\Omega \tau_{N}=1$ is presented in Fig. 2 also exemplifying the quantum effects and showing a pronounced deviation of the quantum SNR curves from the corresponding classical results (up to several orders of magnitude at low temperatures). Meanwhile, we recall that in the classical limit, $S \rightarrow \infty$, and for $\sigma \gg 1, \lambda_{1} \approx \tau_{N} 2 \sigma^{3 / 2} e^{-\sigma} / \sqrt{\pi}$ (Ref. 14) is exponentially small in $\sigma$ and so decreases rapidly as the system is cooled, while all other eigenvalues of the system matrix $\lambda_{k}$ have a nonexponential dependence on $\sigma{ }^{13}$ Hence, at any finite frequency $\Omega$ (i.e., outside the adiabatic limit), the ratio $\Omega / \lambda_{1}$ tends to infinity with decreasing temperature, $T \rightarrow 0$, even at very low frequencies since the interwell transition is almost "frozen out." In spite of this, the spin, although confined to a particular potential well, is not yet completely immobilized and can still take part in intrawell motion. Thus if $\Omega \neq 0, R_{\Omega} \rightarrow$ const as $\sigma \gg 1$ (see Fig. 2). The SNR function $R_{\Omega}$ vs the dimensionless frequency $\Omega \tau_{N}$ is presented in Fig. 3 also illustrating the strong quantum effects for small $S<20$. Here the SNR curves are monotonically increasing functions in $\Omega \tau_{N}$ from the low-frequency limit $R_{0}$ right through to its plateau value $R_{\Omega \rightarrow \infty}$ given by Eqs. (8)-(10).

Here we have studied the magnetic SR of a uniaxial paramagnet of arbitrary spin $S$ for weak spin-bath coupling and Ohmic damping. Thus the correlation time characterizing the

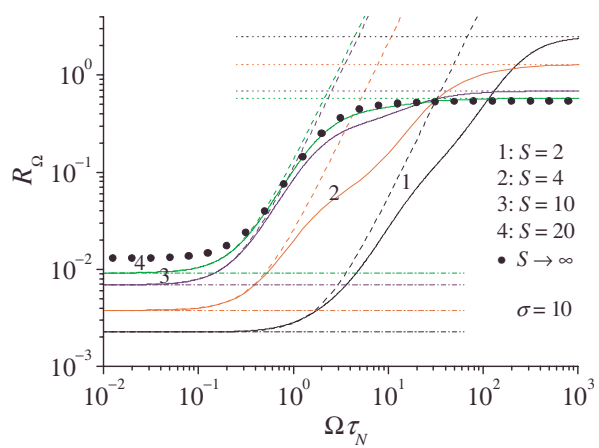

FIG. 3. (Color online) SNR as a function of $\Omega \tau_{N}$ for various $S$ and $\sigma=10$. Solid lines: Eqs. (3) and (5). Dashed lines: the lowfrequency Eqs. (3) and (11). Dashed-dotted and dotted lines: the adiabatic and high-frequency limits, respectively [Eq. (8)]. Filled circles: classical limit, $S \rightarrow \infty$. 
thermal bath is short enough to allow one to approximate the stochastic process originating in it by a Markov process. These approximations may be used in the high-temperature limit, $\beta\left(\varepsilon_{m}-\varepsilon_{m \pm 1}\right) \ll 1$, where $\varepsilon_{m}, \varepsilon_{m \pm 1}$ are adjacent eigenvalues of the energy. The model based on the above approximations describes qualitatively the relaxation in spin systems such as molecular magnets and nanoclusters. ${ }^{17,18}$ Moreover, the model can be regarded ${ }^{18,19}$ as the direct generalization of the Brown theory of relaxation of classical superparamagnetic particles. ${ }^{14}$ In the parameter range, where they fail (e.g., at very low temperatures), more general forms of the density matrix equations must be used (e.g., those treated in Ref. 18).

To conclude, we have estimated the quantum effects in the magnetic SR at the transition from that corresponding to elementary spin relaxation to that pertaining to a giant spin. This allows one to understand the evolution of the magnetic SR from that of molecular magnets $(S \sim 10)$ to nanoclusters $(S \sim 100)$, and to classical superparamagnetic particles $(S \geq 1000)$ and to estimate accurately the value of $S$ (typically in the range of 20-40) at which the crossover to classical superparamagnetic behavior takes place. Furthermore, one may assign a range of validity as a function of the spin size to the classical Brown theory of the magnetization relaxation in superparamagnetic particles with the simplest uniaxial anisotropy. ${ }^{14}$ The relatively elementary calculation outlined above is of particular interest as a basis for future understanding of the SR of spin systems characterized by nonaxially symmetric Hamiltonians commonly used to describe the magnetic properties of molecular magnets and nanoclusters. The SNR for classical superparamagnets having nonaxially symmetric magnetocrystalline anisotropy exhibits a strong intrinsic dependence on the decay rate of the Larmor precession. ${ }^{12}$ This dependence (precession aided relaxation) is due to coupling between longitudinal relaxation and transverse (precessional) modes arising from the lack of axial symmetry. The extension to particular nonaxially symmetric spin systems such as biaxial, cubic, etc. would also allow one to include spin size effects in important applications involving magnetic relaxation in nonaxially symmetric systems, where tunneling in the presence of a transverse field influences the behavior of the reversal time, the switching and hysteresis curves, etc. We also remark that the spin size effects in the nonlinear magnetic SR can be treated by generalizing to the quantum case the nonlinear response theory for classical spins driven by a strong ac field. ${ }^{24}$

This work was emanated from research conducted with the financial support of FP7-PEOPLE-Marie Curie Actions (Project No. 230785 NANOMAGNETS).
${ }^{1}$ L. Gammaitoni, P. Hänggi, P. Jung, and F. Marchesoni, Rev. Mod. Phys. 70, 223 (1998).

${ }^{2}$ B. Lindner, J. García-Ojalvo, A. Neiman, and L. SchimanskyGeier, Phys. Rep. 392, 321 (2004).

${ }^{3}$ J. Casado-Pascual, J. Gómez-Ordóñez, and M. Morillo, Chaos 15, 026115 (2005)

${ }^{4}$ R. F. Fox, Phys. Rev. A 39, 4148 (1989).

${ }^{5}$ A. N. Grigorenko, V. I. Konov, and P. I. Nikitin, Pis'ma Zh. Exp. Teor. Fiz. 52, 1182 (1990) [JETP Lett. 52, 593 (1990)].

${ }^{6}$ E. K. Sadykov, J. Phys.: Condens. Matter 4, 3295 (1992).

${ }^{7}$ L. B. Kiss, Z. Gingl, Z. Márton, J. Kertész, F. Moss, G. Schmera, and A. Bulsara, J. Stat. Phys. 70, 451 (1993).

${ }^{8}$ Yu. L. Raikher and V. I. Stepanov, Phys. Rev. B 52, 3493 (1995).

${ }^{9}$ T. F. Ricci and C. Scherer, J. Stat. Phys. 86, 803 (1997).

${ }^{10}$ Yu. L. Raikher, V. I. Stepanov, and P. C. Fannin, J. Magn. Magn. Mater. 258-259, 369 (2003).

${ }^{11}$ Yu. L. Raikher and V. I. Stepanov, Phys. Rev. Lett. 86, 1923 (2001).

${ }^{12}$ Y. P. Kalmykov, Yu. L. Raikher, W. T. Coffey, and S. V. Titov, Phys. Rev. B 71, 012415 (2005); Fiz. Tverd. Tela (St. Petersburg) 47, 2232 (2005) [Phys. Solid State 47, 2325 (2005)].

${ }^{13}$ W. T. Coffey, Yu. P. Kalmykov, and J. T. Waldron, The Langevin Equation, 2nd ed. (World Scientific, Singapore, 2004).
${ }^{14}$ W. F. Brown, Jr., Phys. Rev. 130, 1677 (1963).

${ }^{15}$ M. Grifoni, L. Hartmann, S. Berchtold, and P. Hänggi, Phys. Rev. E 53, 5890 (1996); P. Reimann and P. Hänggi, Chaos 8, 629 (1998); I. Goychuk and P. Hänggi, Phys. Rev. E 59, 5137 (1999).

${ }^{16}$ I. Goychuk, J. Casado-Pascual, M. Morillo, J. Lehmann, and P. Hänggi, Phys. Rev. Lett. 97, 210601 (2006).

${ }^{17}$ D. A. Garanin, Phys. Rev. E 55, 2569 (1997).

${ }^{18}$ J. L. García-Palacios and D. Zueco, J. Phys. A 39, 13243 (2006); D. Zueco and J. L. García-Palacios, Phys. Rev. B 73, 104448 (2006).

${ }^{19}$ Y. P. Kalmykov, S. V. Titov, and W. T. Coffey, Phys. Rev. B 81, 094432 (2010); J. Stat. Phys. 131, 969 (2008).

${ }^{20}$ W. T. Coffey, D. S. F. Crothers, Yu. P. Kalmykov, and J. T. Waldron, Phys. Rev. B 51, 15947 (1995).

${ }^{21}$ D. A. Garanin, Phys. Rev. E 54, 3250 (1996).

${ }^{22}$ Yu. P. Kalmykov, W. T. Coffey, and S. V. Titov, J. Magn. Magn. Mater. 265, 44 (2003).

${ }^{23}$ R. Kubo, M. Toda, and N. Hashitsume, Statistical Physics II: Nonequilibrium Statistical Mechanics, 2nd ed. (Springer, Berlin, 1991).

${ }^{24}$ P. M. Déjardin and Yu. P. Kalmykov, J. Appl. Phys. 106, 123908 (2009). 